Cuestiones de sociología

ISSN: 2346-8904

publicaciones@fahce.unlp.edu.ar

Universidad Nacional de La Plata

Argentina

\title{
Betina Freidin (coordinadora). Cuidar la salud. Mandatos culturales y prácticas cotidianas de la clase media en Buenos Aires
}

Coniglio, Jimena

Betina Freidin (coordinadora). Cuidar la salud. Mandatos culturales y prácticas cotidianas de la clase media en Buenos Aires

Cuestiones de sociología, núm. 22, 2020

Universidad Nacional de La Plata, Argentina

DOI: https://doi.org/10.24215/23468904e097

Atribución no comercial compartir igual (CC BY-NC-SA) 4.0 


\title{
Betina Freidin (coordinadora). Cuidar la salud. Mandatos culturales y prácticas cotidianas de la clase media en Buenos Aires
}

\author{
Jimena Coniglio \\ Facultad de Humanidades y Ciencias de la Educación | \\ Universidad Nacional de La Plata, Argentina \\ jime.coniglio@gmail.com
}

DOI: https://doi.org/10.24215/23468904e097

El libro es resultado de una investigación sobre los sentidos que un grupo de mujeres y varones de clase media de Buenos Aires le otorgan al imperativo de cuidado de la salud. Fue realizada por el equipo de trabajo integrado por Matías Ballesteros, Mercedes Krause, Pablo Borda, Julia Bonetto, y coordinado por Betina Freidin. Consta de una introducción y seis capítulos temáticos. La perspectiva metodológica es de corte cualitativo, de tipo interpretativo, utilizando la herramienta de grupos focalizados para explorar las interpretaciones y experiencias sobre distintos temas vinculados a las concepciones de salud y bienestar emocional, cuidado médico preventivo, conocimiento y utilización de medicinas alternativas, prácticas alimentarias y actividad física. En el capítulo uno "El trabajo de campo con los grupos focalizados" (Freidin, Ballesteros, Krause) se mencionan las decisiones metodológicas del trabajo de campo. Se realizaron 8 grupos con varones y mujeres de clase media, durante el año 2013 en el Área Metropolitana de Buenos Aires.

En el capítulo dos "Buscando bienestar y equilibrio emocional ante los malestares actuales" (Borda) el autor pone el foco en la relevancia que adquiere el mandato de cuidado de la salud en relación a búsqueda del bienestar y el equilibrio emocional. Uno de los rasgos culturales característicos de las sociedades occidentales es el principio de la expansión personal, el cual promueve un tipo de sujeto predispuesto a descifrarse a sí mismo bajo un ideal de vida saludable. La proliferación de las técnicas terapéuticas orientadas al cuidado de sí evidencian que la divulgación y diversificación del lenguaje psicológico se ha entrelazado con los imperativos culturales contemporáneos de la búsqueda de la felicidad y el bienestar ampliado, la confianza en el potencial interior y la creencia en la capacidad de autoperfeccionamiento, configurando una oferta terapéutica que abarca tanto las técnicas psicoterapéuticas como una diversidad de prácticas de cuidado terapéutico que articula de forma ecléctica las premisas del discurso de autoayuda, los aportes filosóficos del Movimiento New Age y la espiritualidad. Particularmente en Argentina, la institucionalización del psicoanálisis y la gran difusión del mismo en los sectores sociales medios y altos urbanos, generó una identificación con la figura del psicoanalista y la práctica clínica. Como resultado del análisis de los grupos focalizados, en líneas generales, los/as participantes coinciden en comprender la salud como un estado de equilibrio entre las dimensiones corporales y las psicoemocionales, pero algunos hacen énfasis en la preeminencia de lo mental sobre lo corporal. Este estado de equilibrio, se expresaría en algunos/as participantes, como un bienestar general que permitiría el desarrollo de una vida plena, armoniosa y saludable; el logro de ese estado es el resultado de una búsqueda personal, de un trabajo reflexivo sobre sí mismo. A su vez, se analizan una serie de prácticas desarrolladas por los/as participantes que el autor nomina "prácticas de salud emocional anti estrés", como dedicar tiempo a las actividades de ocio, esparcimiento y recreación. Estas se emparentan con los imperativos culturales tendientes a afianzar los gustos subjetivos, a encontrar tiempo para uno mismo.

En el capítulo tres "El cuidado de la salud y la percepción de riesgos: género, ciclo vital y experiencias biográficas" (Freidin y Krause) las autoras analizan las actitudes de los/as participantes de los grupos focalizados frente al cuidado médico preventivo, atendiendo a las diferencias de género en su interrelación con los momentos del ciclo vital y las experiencias familiares con la enfermedad. En un contexto sociocultural 
en el que se ha depositado una gran responsabilidad individual para disminuir el riesgo de enfermedad, se espera que los sujetos desarrollen hábitos de vida saludables y se realicen monitoreos médicos. Se observó cómo algunos/as participantes describieron las prácticas de cuidado como propias de cada género, reproduciendo las representaciones binarias dominantes. Entre las jóvenes, la principal motivación para realizar prácticas alimentarias y corporales es estética. Respecto a la importancia del monitoreo médico periódico, los/as participantes evalúan las circunstancias en cada caso, de acuerdo a la gravedad percibida de los síntomas por ellos/as mismos/as o su entorno familiar. También se abordó, la cuestión del escepticismo y la desconfianza respecto a la consulta médica; se observó que la autoridad médica es central en las estrategias de cuidado de los/as participantes, aunque en la decisión de la consulta tanto preventiva como curativa relativa a malestares menores opera una jerarquía de utilización de recursos de cuidado, incluyendo la autoatención y la automedicación como prácticas habituales, y en algunos casos, la preferencia por recurrir a especialistas de las medicinas alternativas. Se destaca el rol de las mujeres/madres como las que generalmente articulan distintos saberes y aprendizajes a lo largo del tiempo para resolver episodios de salud en el grupo familiar. También, se incluyó en este capítulo el papel de la información digital; los/as participantes pusieron en cuestión los beneficios de la abundante información médica disponible en internet, especialmente en la instancia previa (e incluso paralela) a la consulta con el/la especialista.

En el capítulo cuatro "Las medicinas alternativas: conocimiento, experiencias de utilización y lógicas de 'desuso'” (Freidin) se indagan las visiones y las modalidades de uso de las medicinas alternativas y complementarias (MAC). Se considera a las MAC a aquellos enfoques médicos y terapias que no están integradas en el sistema de salud, cuyas prácticas de cuidado y productos se diferencian de la medicina alopática. Aunque el campo específico de las MAC es difícil de delimitar, se indagó en este capítulo por el conocimiento y el uso, teniendo en cuenta la familiaridad con ellas y la jerarquía de credibilidad otorgada a los distintos saberes y ofertas terapéuticas. En consonancia con los hallazgos del capítulo anterior, aparece la cuestión de género en relación al uso de MAC, en efecto, las mujeres de clase media de mediana edad y con hijos son las que tienen mayor experiencia en utilización de las mismas; la decisión de recurrir a ellas, para distintas dolencias y necesidades de cuidado, se vio orientada tanto por visiones más críticas hacia la biomedicina como por razones pragmáticas. El uso de las MAC se relaciona fundamentalmente con el tratamiento de enfermedades, especialmente para condiciones crónicas, buscando evitar el uso de fármacos. En los grupos de discusión se distinguieron usuarios más experimentados y estables, y usuarios circunstanciales o de uso excepcional (que fueron fundamentalmente hombres). Además, se analizó la lógica del "desuso" de las MAC a partir del descreimiento frente a la legitimidad científica de la biomedicina; como factores disuasivos se consideraron el costo de las consultas con especialistas alternativos, así como también la cuestión de la eficacia simbólica basada en la "creencia" que muchos/as de los/as no usuarios les atribuyen, asociadas asimismo a las barreras morales construidas entre los/as distintos/as especialistas del diversificado campo del cuidado alternativo.

En el capítulo cinco "Alimentación saludable y vida cotidiana” (Freidin y Bonetto) se analiza cómo los/ as participantes de los grupos de discusión se posicionan ante los discursos expertos y publicitarios de la alimentación saludable, cuya atención se articula con el ideal de alcanzar un cuerpo joven, delgado y atractivo a través del esfuerzo personal. La preocupación por la alimentación es integral al mandato sociocultural del "imperativo de la salud" que promueve la responsabilidad individual; esta cuestión tuvo especial resonancia en las clases sociales altas y medias, con mayor acceso a la información sobre regímenes alimentarios promovidos como saludables y mayor capacidad de movilizar recursos para implementarlos. Aquí también entran en juego las lógicas de negociación entre el placer personal que proporciona la comida y la preocupación estética por mantener el cuerpo en forma. En las discusiones con los grupos aparecen distintas preocupaciones referidas a la temática: la percepción de riesgos en torno al acto alimenticio, las cuestiones ético-ambientales en relación a la industria alimenticia, la alimentación saludable (el consumo light y la articulación entre disciplina y placer), y la alimentación en el trabajo en relación de dependencia (como una cuestión que limita las posibilidades de 
seguir una dieta saludable). También en este capítulo aparece la perspectiva de género; en efecto, alimentar saludablemente al grupo familiar suele ser una tarea femenina invisibilizada que, además de tiempo, requiere ocuparse del menú y de la provisión. La preocupación por la alimentación de los/as hijos/as no es vivida de la misma manera por los varones-padres que por las mujeres-madres, reproduciendo los roles de género dominantes, ya que son estas últimas las que cargan con la responsabilidad moral y la tarea cotidiana de alimentar apropiadamente a los/as integrantes del grupo doméstico.

En el capítulo seis (y último) "Entre la compensación, el placer, el bienestar psicofísico y la experiencia del presente: la práctica de actividad física entre sectores de clase media” (Ballesteros) el autor aborda la actividad física en relación al cuidado de la salud psicofísica. A pesar de que las posibilidades para realizar ejercicio físico están fuertemente estratificadas, el contexto sociocultural enfatiza en la responsabilidad individual para realizarlo, acompañada de una carga moral sobre los comportamientos. En los grupos de discusión las motivaciones para la realización están vinculadas con los beneficios específicos para la salud (o frente a una necesidad de salud), el bienestar general, la estética corporal y las lógicas compensatorias (como balance para contrarrestar comportamientos "dañinos" para el cuerpo sobre todo en los grupos de jóvenes) siendo estas complementarias. Pero además aparecen motivaciones que se relacionan con el disfrute, la sociabilidad e interacción social, la liberación de tensiones (estrategia de "descarga" antiestrés) y el sentido del logro. En los grupos, para algunos/as la actividad física aparece como un mandato moral, cuyo "no cumplimento" es vivido con culpa. Las trabas para la realización aparecen asociadas a la falta de tiempo, la dificultad de encontrar un grupo para realizar una práctica y la falta de ofertas y espacios donde realizarla. En los grupos de mujeres con hijos, aparece un conflicto entre el disponer del tiempo personal para la actividad física con las responsabilidades familiares, domésticas y laborales.

En síntesis, el trabajo de los/as autores/as se presenta como una importante contribución al analizar las prácticas de cuidado como elementos prominentes en las sociedades modernas en los capítulos temáticos, dando cuenta de la perspectiva de género como una cuestión que atraviesa los modos de cuidar, a la vez que utiliza la herramienta de los grupos focalizados para la recolección de los datos cualitativos.

\section{BY-NC-SA}

